\title{
ENHANCED AIR OPERATIONS FOR GROUND SITUATIONAL AWARENESS
}

\author{
Erik Blasch, AFRL/RIEA, Rome, $N Y$ \\ Zhonghai Wang, Dan Shen, Genshe Chen, Information Fusion Technology, MD \\ Haibin Ling, Temple University, Philadelphia, PA
}

\begin{abstract}
Future digital avionics systems will work in complex and cluttered environments which require systems engineering solutions for such applications as airport ground surface management. In this paper, we highlight the use of a L1 video tracker for monitoring activities at an airport. We present methods of information fusion, entity detection, and activity analysis using airport videos for runway detection and terminal events. For coordinated airport security, automated ground surveillance enhances efficient and safe maneuvers for unmanned air vehicles (UAVs) and unmanned ground vehicles (UGVs) operation at airport environments.
\end{abstract}

Keywords: Airport Security, Ground Surface Management, Video Surveillance

\section{Introduction}

Airport security is critical for personnel safety, efficient vehicle coordination, and effective airspace utilization. The Next Generation Air Transportation System (NextGen) and Single European Sky Air Traffic Management Research (SESAR) seek methods for safe and efficient air and ground operations [1]. Figure 1 shows many aspects of airport security including air traffic control, security operations, tower visualization, and ground-based surveillance. While airport security is typically associated with manned aircraft, future scenarios could include unmanned air vehicles (UAVs) take-off and landings as well as unmanned ground vehicles (UGVs) to monitor ground activities. Thus, it is important to consider automated video exploitation methods for ground surface security operations to alert Air Traffic Controllers (ATC) of impending collisions and security threats.

A key future technology for aviation is NextGen air traffic control [2]. Typically, an airport is monitored from radar and video for airplane safety, ground operations, and security. In most cases, an ATC monitors events to make sure operations are proceeding as desired. Analogously, there are emerging situations in which UAVs are operating in airport environments in which there is a need to coordinate safe flight (e.g., take-off and landings) using the same transportation routes (e.g., runways). In this study, we address a future topic that looks at autonomously monitoring transportation routes at an airport such that there is an advantageous use of automatic video surveillance that can cue ATC operators on possible collisions of ground transportation systems.

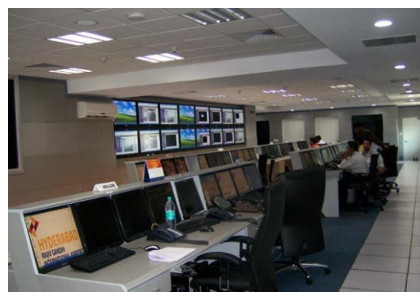

(a)

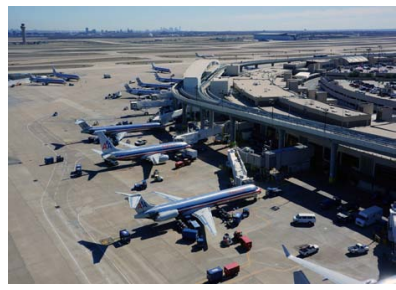

(c)

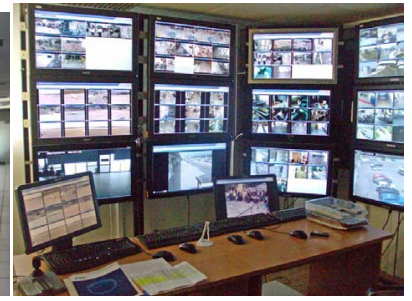

(b)

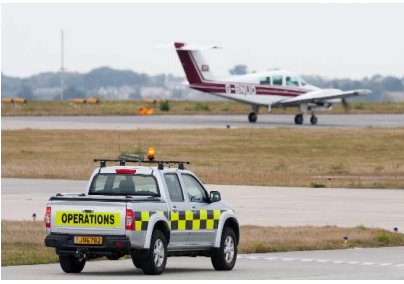

(d)
Figure 1. ATC Activates for Airport Security: (a) Controller, (B) Camera Images, (C) Aerial Images, (D) Ground Cameras

Automated surveilliance of ground surface operations at an airport includes information fusion $[3,4]$ of multile sensors. Surface management also requires collaborative automation [5] and integrated displays [6]. These automated systems must be integrated with airport transportation methods for safe distance coordination of vehicles and the people that operate them [7]. A common discussion includes runway incursion prevention for safe operations [8]. Recent methods include cooridnation of ground operations with airport navigation [9], distributed 
management [10], and innovative approaches to terminal routing [11].

In order to explore advances in automated video tracking for safe airport operations, we seek information fusion methods for distributed management of surveillance techniques. In Section 2, we highlight information fusion and Section 3 activity analysis. Section 4 details the video tracking method. Section 5 presents results for assessment of safe airport taxi crossing where vehicles cross the runway as well as methods for person-vehicle-object detection for airport terminal operations. Section 6 provides discussion and conclusions.

\section{Information Fusion}

Airport surveillance can include full-motion video (FMV) [12] and wide-area motion imagery (WAMI) [13] for semantic scene labeling. Here we utilize information fusion tracking for person-objectvehicle-facility (POVF) relational activity labeling. Activity detection can alert an ATC operator of impending threats [14], reduce the data overload [15] and detect anomalies from social and cultural airport norms [16]. Thus, we seek analytical and computational methods of enhanced exploitation from video to assist and augment humans in rapidly assessing activity analysis for acquiring situation awareness [17] and threat analysis [18]. We use the , context, and communications [19] paradigm for airport operations (see Table 1).

Table 1. Elements of Cues, Contexts, and Channels

\begin{tabular}{|l|l|l|l|}
\hline & Cues & Contexts & Channels \\
\hline Vision & $\begin{array}{l}\text { Feature } \\
\text { extraction } \\
\text { and } \\
\text { processing }\end{array}$ & $\begin{array}{l}\text { Culture- } \\
\text { based } \\
\text { exploitation } \\
\text { and search } \\
\text { annotation }\end{array}$ & $\begin{array}{l}\text { Media } \\
\text { (video) for } \\
\text { dissemination } \\
\text { of content }\end{array}$ \\
\hline Tracks & $\begin{array}{l}\text { Geo- } \\
\text { location }\end{array}$ & Semantic & Network \\
\hline Semantics & Grammars & Linguistic & Annotations \\
\hline Situation & Perspective & Knowledge & Interaction \\
\hline User & $\begin{array}{l}\text { Scene } \\
\text { analysis }\end{array}$ & $\begin{array}{l}\text { Cooperative } \\
\text { data }\end{array}$ & $\begin{array}{l}\text { Knowledge } \\
\text { transfer }\end{array}$ \\
\hline
\end{tabular}

Figure 1 shows examples of multi-data collection for airport operations. Data fusion requires collecting data, filtering the relevant information, and providing cues for user refinement [20]. Organizing all sources of information requires distributed database information coordination [21]. Filtering relevant data is important when there are many hours of video where there is no activity of interest and users are constrained by numerous simultaneous actions. Exploitation analysis, such as activity recognition, provides cues on impending threats [22]. Current challenges in information fusion include situation and threat awareness [23], such as alerting an ATC to collisions of entities (airplanes, people, objects, and vehicles).

Context is provided by normal user operations such as an airport. Using a Cognitive ObserveOrient-Decide-Act (OODA) processing loop [24], machine-human interaction for a UAV/UGV video provides efficient control using the robotic operating system [25]. The controller provides alerts, representations and context. However, not all processing can be done by the machine as there are data uncertainties [26] associated with the information channels. Thus, shared awareness between the man and machine requires judgment for situation understanding [27, 28, 29]. Exploiting channels and context of activities of importance [30] context can guide which cues to report for airport safety.

Figure 2 demonstrates an effects-based operations (EBO) information fusion paradigm. The Data Fusion Information Group (DFIG) process model [31] Levels (L) are indicated on the left, over cognitive, information, and physical domains. The physical domain includes data characterization (L0) and object assessment (L1). The information domain builds on the data from the physical domain for situation (L2) and impact (L3) assessment with sensor management (L4) [32]. The cognitive domain includes user (L5) and mission (L6) refinement [33]. High-Level Information Fusion (L2-L6) builds on Low-level (L0-L1) analysis which utilizes an ontology [34, 35, 36] of activities. Object and event detection have been subjects of analysis for the last decade from which we build on these strategies for measures of effectiveness [37] in activity reporting. 


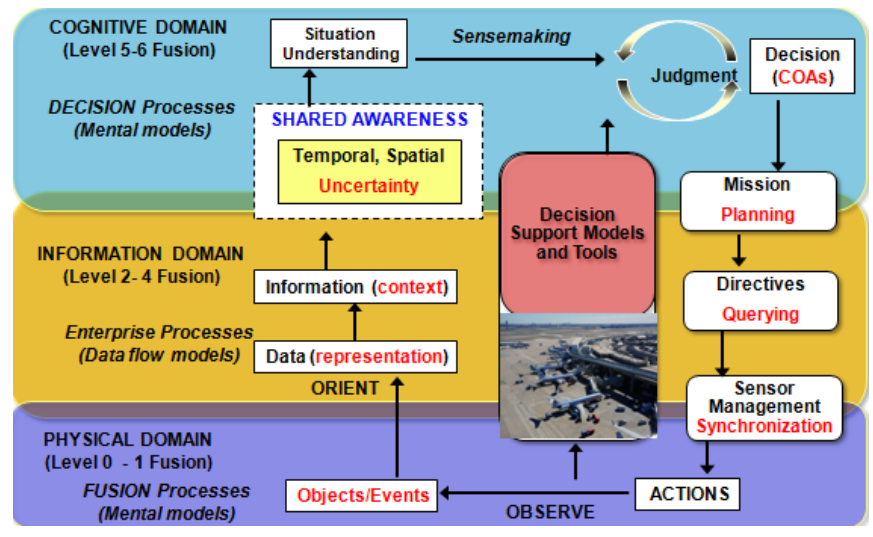

Figure 2. Effects-Based Information Fusion Reasoning

\section{Video Tracking}

Video tracking has been widely studied so we focus on activity analysis for context assessment. Standard methods of appearance-based (e.g., color, structure) analysis supports the detection of people, airplanes, vehicles, and airport terminals.

\subsection{Scene Analysis}

One approach is to bridge the gap between lowlevel image features and high-level semantic representations [38]. An example they use is news information that extracts keywords from the news broadcast and links them to the features and objects in the video (e.g., vehicles, people clothing). Using a semantic database, WordNet, they captured the relevant text attributes in the context of video. Scene analysis through context-guided search between object and event detection and recognition using audio (linguistic semantics) and video (image features) is demonstrated.

Semantic modeling can be extended by mapping vision to language using signal-to-fusion through Bayes Nets [39] that was applied for automatically annotating broadcast video. Three types of objects in the video were used, namely people, vehicles, and stationary scene elements. The semantic analysis included objects (e.g., person), verbs (e.g., motion), pruning (scene descriptors), and attributes (fast, slow, north). Results demonstrated the ability to identify relevant videos related to semantic concepts.

\subsection{Learning Relationship Patterns}

Pattern analysis includes many forms of data aggregation such as motion information detected from tracking. Chan et al. [40], use a Hidden Markov Model (HMM) to determine complex events based on tracks for an airport scenario. They use a spatial distance as a semantic relationship "close" to link when a vehicle is close to the plane and duration times to estimate loading and unloading. Additional developments [41] include a Dynamic Bayesian Network as a generalization to a HMM to model events, and when tracks are fragmented can better link objects with observed recognition (or identity). Using semantic labels for events (e.g., truck is close to plane); then the tracklets can be combined for a complex event (e.g., plane refueling) through analysis of an event log-likelihood score.

Pixel level pdfs of object speed and size from the tracks can be modeled as a multivariate Gaussian Mixture Model (GMM) of the motion (destination location and transition time) and the size (width and height) parameters of the objects at that location [42]. Track outputs, with unsupervised expectationmaximization-based learning of every GMM and scene modeling detect local as well as global anomalies in object tracks. Other techniques include clustering [43, 44], histogram of gradients [45, 46], and bag of visual words [47] that can label pattern of life [48] activities.

\subsection{Detecting People Versus Vehicles}

The semantic differences between people and vehicles can be modeled using their size and movement characteristics. Key papers focus on human activities [49, 50] versus vehicle activities [51]. While single event activity recognition has a plethora of research, ontologies for human-vehicle relationships are still needed for safe airport operations. Using notional patterns, a vehicle path supports normal operations (e.g., luggage carrier). All of these patterns, whether people, vehicles, or airplanes can be used for normalcy modeling (e.g., runway taxis) and anomaly detection for situation awareness.

\subsection{Evaluation}

Future developments require objective assessment of video trackers, score fusion, and semantic labeling of video clips. Such examples include audio-visual features text labeling over vehicles [52] and people activities for video indexing and retrieval [53]. To determine the people-vehicle- 
airplane interactions requires analysis of the operating conditions of the sensor, target, and environment [54]. For example, the sensor (video) looks for targets (vehicles) in the environment (runways). The use of context, [55], from the machine or human [56] helps refine and highlight activities in the video.

\section{Event/Activity Detection}

Our goal is to use detected events for activity analysis from video for association, relationship analysis, mining, exploration, and discovery (ARMED) techniques. We seek analysis of activities and events as defined below:

Activity: A specified pursuit in which a person partakes. A mental function of an organism or organization (Webster)

Event: the final or postulated outcome, condition, or determination of occurrence of an activity or experiment over space-time (Webster)

An activity relates to an actor (e.g., person) doing a function or action; while an event is the outcome of the occurrence of an activity. The inverse is that a detected event requires a causal relation to an activity. Causality results from the duration of the activity (e. g, person loading an airplane) and the final event (e.g., person loads luggage into airplane). Use cases include complex tracks and surveillance. For surveillance, many events might exist that don't have activities that require actionable response. A person walking is an activity; whereas a person walking (entering) an area of regard is an important event. For a person loading an airplane, it is an activity, but a specific person at an airplane is an event. The key is to nominate events (e.g., spatial threats) that require space-time correlation for activities analysis. In some cases, the designated objects/places of interest cue activity recognition for event detection. However, the reverse is not true in that all events don't require activity labeling (e.g., stationary airplane).

For the fusion of networked cameras, there is a need for correlation and information management $[57,58]$. To manage the multiple sensors, there is needed both the ability and the policies associated with being able to combine sensor data. Policies [59] of different sensor collections might preclude the ability to actually fuse the data (such as collected from different airport assets). We assume for this paper that airport videos, collected from air and ground surveillance cameras, can be shared between airport facilities.

\subsection{Activity Schema for Alerting}

Using the video analysis for target tracking [60], event detection [61], activity recognition [62, 63], and scene analysis [64] requires a common schema policy. The ontology content should use a common message passing schema, with fields such as at $<$ time $><$ place $><$ pers, veh, obj $\}$, qty $><$ activity $>$ that are available through video extraction. Using the schema, results from distributed video tracking [65], sparse scenes [66], person-vehicle interactions [67], and person-vehicle-object-facility models [68], can be updated and reported to ATC airport operations.

For the case of the airport ground surveillance, using a relevant scenario of real world data, many context situations arise. The goal then is to provide links (entity relationships) to automation (user coordination) for airport operations. Video fusion methods need to accommodate different policies and be predictive in the analysis to determine which sensors and data would support for association. Predictive analysis is like discovery. Both the stored images and real-time video exploitation require consistent ontology representations for causal, coincident, and irrelevant probability scoring of activities given situational context.

\subsection{Context}

Leveraging developments from computer vision, numerous contextual issues have been reported that support airport surveillance. FMV analysis includes image stabilization $[69,70]$, multiple objects and group tracking $[71,72,73]$, road assessment and tracking [74, 75], feature selection [76, 77] and contextual tracking [78, 79]. Context aids in persistent tracking [80, 81], target localization [82], object detection [83, 84, 85] and target association [86]. Because of the numerous objects and their dynamic movements, there are opportunities for linear tracking (e.g. runways), but also there is a need for nonlinear track evaluation [87, 88], such as the randomized unscented transform (RUT) filter [89] for refinement of track accuracy. Here we focus on the nonlinear L1 tracker for event detetcion and activity alerting. 


\section{L1 Tracking Framework}

The L1 particle filter tracker [90, 91, 92, 93] takes advantages of the sparse representation and compressive sensing techniques. The original L1 tracker was computationally expensive due to the intensive computation of L1 solutions. But several techniques have been developed to speed up the process for near real time analysis.

\subsection{Particle Filter}

The particle filter provides an estimate of posterior distribution of random variables related to Markov chain. In visual tracking, it gives an important tool for estimating the target of next frame without knowing the concrete observation probability. It consists of two steps: prediction and update. Specially, at the frame $t$, denote $x_{t}$ which describes the location and the shape of the target, $y_{1: t-1}=\left\{y_{1}, y_{2}, \cdots, y_{t-1}\right\}$ denotes the observation of the target from the first frame to the frame $t-1$. Particle filter precedes two steps with following two probabilities:

$$
\begin{gathered}
p\left(x_{t} \mid y_{1: t-1}\right)=\int p\left(x_{t} \mid x_{t-1}\right) p\left(x_{t-1} \mid y_{1: t-1}\right) d x_{t-1} \\
p\left(x_{t} \mid y_{1: t}\right)=\frac{p\left(y_{t} \mid x_{t}\right) p\left(x_{t} \mid y_{1: t-1}\right)}{p\left(y_{t} \mid y_{1: t-1}\right)}
\end{gathered}
$$

The optimal state for frame $t$ is obtained according to the maximal approximate posterior probability: $x_{t}^{*}=\arg \max _{x} p\left(x \mid y_{1: t}\right)$.

The posterior probability is approximated by using finite samples $S_{t}=\left\{x_{t}^{l}, x_{t}^{2}, \cdots, x^{N}{ }_{t}\right\}$ with different weights $W=\left\{w^{1}{ }_{t}, w^{2}{ }_{t}, \cdots, w^{N}\right\}$ where $N$ is the number of samples. The samples are generated by sequential importance distribution $\Pi\left(x_{t} \mid y_{1: t}, x_{1: t-1}\right)$ and weights are updated by:

$$
w_{t}^{i} \propto w_{t-1}^{i} \frac{p\left(y_{t} \mid x_{t}^{i}\right) p\left(x_{t}^{i} \mid x_{t-1}^{i}\right)}{\prod\left(x_{t} \mid y_{1: t}, x_{1: t-1}\right)}
$$

When $\Pi\left(x_{t} \mid y_{1: t} x_{1: t-1}\right)=p\left(x_{t} \mid x_{t-1}\right)$, the above equation takes a simplified form $w_{t}^{i} \propto$ $w_{t-1}^{i} p\left(y_{t} \mid x_{t}^{i}\right)$. Then, the weights of some particles maybe keep increasing and fall into the degeneracy case. To avoid such a case, in each step, a resampling strategy is used to generate samples with equal weights according to previous sample weights distribution.

\subsection{Sparse Representation}

The sparse representation model aims at calculating the observation likelihood for sample state $x_{t}$, i.e. $p\left(z_{t} \mid x_{t}\right)$. At the frame $t$, given the target template set $T_{t}=\left[t_{t}{ }_{t}, t_{t}^{2}, \cdots, t_{t}^{n}\right]$, let $S_{t}=\left\{x_{t}{ }_{t}, x_{t}^{2}, \cdots, x^{N}{ }_{t}\right\}$ denote the sampled states and let $O_{t}=\left\{y_{{ }_{b}}{ }_{b} y_{t}{ }_{t}, \cdots, y^{N}{ }_{t}\right\}$ denote the corresponding candidate target patch in target template space. The sparse representation model is then:

$$
y_{t}^{i}=T_{t} a_{T}^{i}+I a_{I}^{i}, \forall y_{t}^{i} \in O_{t},
$$

where $I$ is the trivial template set (identity matrix) and $a_{t}^{i}=\left[a_{T}^{i} ; a_{I}^{i}\right]$ is sparse in general. Additionally, nonnegative constraints are imposed on $a_{T}^{i}$ for the robustness of the L1 tracker. Consequently, for each candidate target patch $\mathrm{y}_{\mathrm{t}}^{\mathrm{i}}$, the sparse representation of $\mathrm{y}_{\mathrm{t}}^{\mathrm{i}}$ can be found via solving the following L1-norm related minimization with nonnegative constraints:

$$
\min _{a} \frac{1}{2}\left\|y_{t}^{i}-A a\right\|_{2}^{2}+\lambda\|a\|_{1}, a \geqslant 0
$$

where $A=\left[T_{t}, I,-I\right]$.

Finally, the observation likelihood of state $x_{t}^{i}$ is given as:

$$
p\left(z_{t} \mid x_{t}^{i}\right)=\frac{1}{\Gamma} \exp \left\{-a\left\|y_{t}^{i}-T_{t} c_{T}^{i}\right\|_{2}^{2}\right\},
$$

where $\alpha$ is a constant controlling the shape of the Gaussian kernel, $\Gamma$ is a normal factor and $c_{T}^{i}$ is the minimizer of the L1-norm minimization restricted to $T_{t}$. Then, the optimal state $x_{t}^{*}$ of frame $t$ is obtained by:

$$
x_{t}^{*}=\arg \max _{x_{t}^{i} \in S_{t}} p\left(z_{t} \mid x_{t}^{i}\right) .
$$

In addition, a template update scheme is adopted to overcome pose and illumination changes.

\subsection{Modified Version for Occlusions and Noise}

There are two types of dictionary templates: target templates and trivial templates. The target templates are updated dynamically for representing target objects during the tracking process. The trivial templates (identity matrix $I$ ) is for representing occlusions, background and noise. However, since parts of objects may also be represented by the trivial templates, the region detected by the original tracker sometimes does not fit the target very accurately.

We use a modified version for improving tracking accuracy. The new model is based on the following observation. When there are no occlusions, 
the target in the next frame should be well approximated by a sparse linear combination of target templates with a small residual. Thus, the energy of the coefficients in $a$ associate with trivial templates, or trivial coefficients, should be small. On the other hand, when there exist noticeable occlusions, the target in the next frame cannot be well approximated by any sparse linear combination of target templates, the large residual (corresponding to occlusions, background and noise in an ideal situation) will be compensated by the part from the trivial templates, which leads to a large energy of the trivial coefficients. The minimization is obviously not optimal since it does not differentiate these two cases.

To optimize the usage of the trivial templates in the tracking, we need to adaptively control the energy of the trivial coefficients. That is, when occlusions are negligible, the energy associated with trivial templates should be small. When there are noticeable occlusions, the energy should be allowed to be large. This motivation leads to the following minimization model for L1 tracker:

$\min _{a} \frac{1}{2}\left\|y-A^{\prime} a\right\|_{2}^{2}+\lambda\|a\|_{1}+\frac{\mu_{t}}{2}\left\|a_{I}\right\|_{2}^{2}$, s.t. $a_{T} \geqslant 0$,

where $A^{\prime}=\left[T_{t}, I\right], a=\left[a_{T} ; a_{I}\right]$ are the coefficients associated with target templates and trivial templates respectively, and the parameter $\mu_{t}$ is a parameter to control the energy in trivial templates. In our implementation, the value of $\mu_{t}$ for each state is automatically adjusted using the occlusion detection method. That is, if occlusions are detected, $\mu_{t}=0$; otherwise $\mu_{t}$ is set as some pre-defined constant. The benefit of the additional L2 norm regularization term is that the trivial templates coefficients from minimization are small and lead to better tracking results.

\subsection{Minimum Error Bound}

A minimal error bounding method is proposed to reduce the number of needed L1 minimizations. Actually, the method is based on the following observation:

$$
\left\|T_{t} a-y\right\|_{2}^{2} \geq\left\|T_{t} \hat{a}-y\right\|_{2}^{2}, \forall a \in \mathbb{R}^{N}
$$

where, $\quad \hat{a}=\arg \min _{a}\left\|T_{t} a-y\right\|_{2}^{2}$

Consequently, for any samples $x_{t}^{i}$, its observation likelihood has the following upper bound: $p\left(z_{t} \mid x_{t}^{i}\right)=\frac{1}{\Gamma} \exp \left\{-a\left\|y_{t}^{i}-T_{t} c_{T}^{i}\right\|_{2}^{2}\right\} \triangleq q\left(z_{t} \mid x_{t}^{i}\right)$

where $q\left(y_{t}^{i} \mid x_{t}^{i}\right)$ is the probability upper bound for state $x_{t}^{i}$. It is seen that if $q\left(z_{t} \mid x_{t}\right)<\frac{1}{2 N} \sum_{j=1}^{i-1} p\left(z_{t} \mid x_{t}^{j}\right)$, then the sample $x_{t}^{i}$ will not appear in the resample set. In other words, $x_{t}^{i}$ can be discarded without being processed. Thus, a two stage resample method is used to significantly reduce the number of samples needed in tracking.

\subsection{Accelerated Proximal Gradient (APG)}

The APG method is designed for solving the unconstrained minimization. Thus, we need to convert the constrained minimization model into an unconstrained problem. Let $1 \in R^{N}$ denote the vector with all entries are equal to 1 and let $1^{\mathrm{R}}{ }_{\mathrm{N}+}$ (a) denote the indicator function defined by:

$$
1_{\mathbb{R}_{+}^{N}}(a)=\left\{\begin{array}{c}
0, a \geqslant 0 ; \\
+\infty, \text { otherwise. }
\end{array}\right.
$$

So, the minimization equation in the modified version is equivalent to the following minimization problem:

$$
\arg \min _{a} \frac{1}{2}\left\|y-A^{\prime} a\right\|_{2}^{2}+\lambda\|a\|_{1}+\frac{\mu_{t}}{2}\left\|a_{I}\right\|_{2}^{2}+1_{\mathbb{R}_{+}^{N}}\left(a_{T}\right) .
$$

Then, the APG model will be:

$$
\begin{gathered}
\min F(a)+G(a), \\
F(a)= \\
\frac{1}{2}\left\|y-A^{\prime} a\right\|_{2}^{2}+\lambda 1_{T}^{T} a_{T}+\frac{\mu_{t}}{2}\left\|a_{I}\right\|_{2}^{2}, \\
G(a)=\left\|a_{I}\right\|_{1}+1_{\mathbb{R}_{+}^{N}}\left(a_{T}\right) .
\end{gathered}
$$

To solve the above optimization problem, we use Algorithm 1: 
Algorithm 1: Numerical algorithm for solving minimization

(i) Set $a_{0}=a_{-1}=0 \in \mathbb{R}^{N}$ and set $t_{0}=t_{-1}=1$.

(ii) For $\mathrm{k}=0,1 \ldots$, iterate until convergence

$$
\begin{aligned}
\beta_{k+1} & :=\alpha_{k}+\frac{t_{k-1}-1}{t_{k}}\left(\alpha_{k}-\alpha_{k-1}\right) \\
g_{k+1 \mid T} & :=\beta_{k+1 \mid T}-\left.\left(A^{T}\left(A^{\prime} \beta_{k+1}-y\right)\right)\right|_{T} / L-\lambda 1_{T} ; \\
g_{k+1 \mid I} & :=\beta_{k+1 \mid I}-\left.\left(A^{T T}\left(A \beta_{k+1}-y\right)\right)\right|_{I} / L-\mu \beta_{k+1 \mid I} / L ; \\
\alpha_{k+1 \mid T} & :=\max \left(0, g_{k+1 \mid T}\right) \\
\alpha_{k+1 \mid I} & :=\sum_{\lambda / L}\left(g_{k+1 \mid I}\right) \\
& t_{k+1}:=\left(1+\sqrt{1+4 t_{k}^{2}}\right) / 2 .
\end{aligned}
$$

Then, our final APG-L1 tracker is Algorithm 2.

\subsection{Implementation Details}

The number of target templates is set to 10 . To first initialize the templates, we make very small perturbations around the labeled target area. The typical template height is set to 18 and the width is automatically adjusted according to the labeled target size. The number of particles is set to 600 , and is a balance between computational speed and accuracy. Updating is also a critical part for activity analysis, where a very aggressive or passive strategy will both lead to track drifting. The main criterion to determine the update is the vector angle between the template and the tracked target. A useful setting is when this angle is $>40$, which we regard as a missed track. When the angle is between ${ }^{\circ} 3$ and $40^{\circ}$, we will remove the least matched template and include a new template. When the angle is $<30$ we consider it as accurate tracking and no template needs updating. Since we use aerial video sequences, the targets are considerably small in the image range, thus we may assume they are fixed in size. When we sample the particles, we could then only make perturbations at the position level so that the search dimension is further reduced.

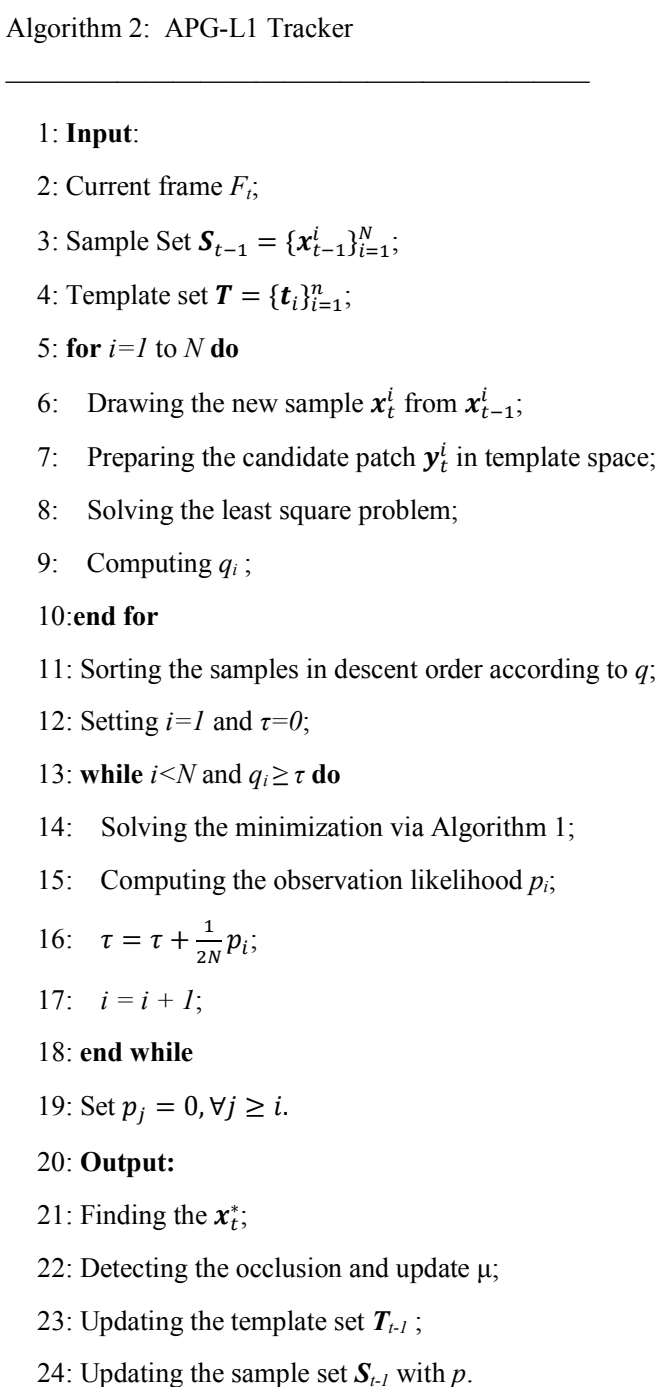

Another method in conjunction with the targetbased template updating is to use a likelihood estimate. From the L1 results, we can refine the estimate based on the likelihood ratio (LR) (which is a normalized method for probabilities) [94]. LR based on the context to refine activity estimates. The use of the likelihood analysis relates to graphical methods that link the actors to activities from contextual events.

\section{Exemplar Results}

We provide two examples for video tracking for airport security. These examples motivate the future operations of UAVs and UGVs with cameras monitoring airport activities. The first case is from the Gibraltar airport, which is one of the only airports 
where the runway intersects a road [95], as shown in Figure 3.

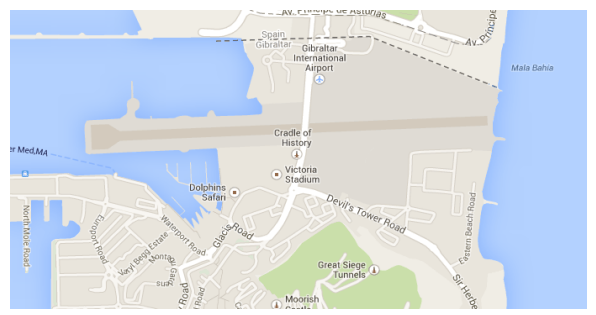

Figure 3. Gibraltar Airport

Video sequences were accessed online from normal operations [96]. Figure 4 shows the case of detecting the movement of vehicles on the road from a surveillance video. Likewise, Figure 5 shows the video detection and tracking of an aircraft take-off. Obviously, having the same viewing coverage, it would be easy to determine the location of vehicles using the road that might be entering the area of the runway. The situation is analogous to UAVs requiring autonomous recognition of surface operations for runway clearance.

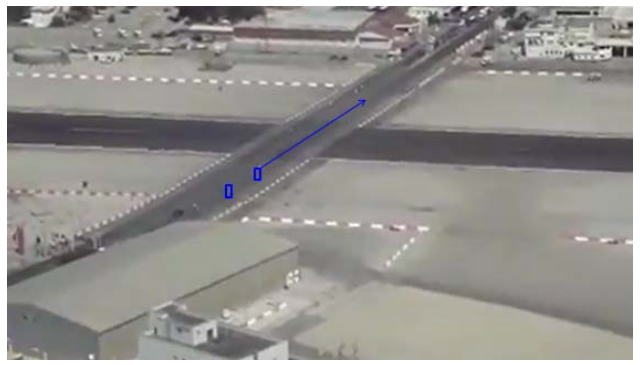

Figure 4. Cars Crossing Runway at Gibraltar Airport

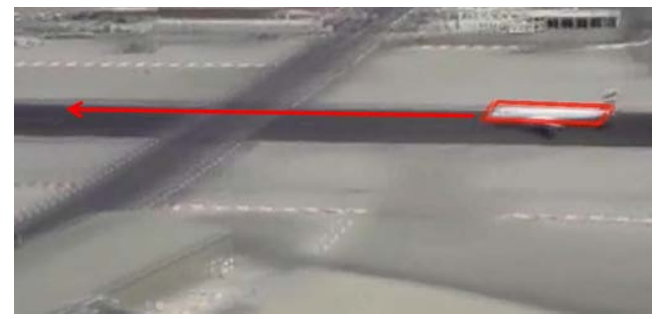

Figure 5. Airplane Detection on Runway

For the second case, we use the Stuttgart Airport with a live webcam [97]. In Figure 6, we show an example of people and vehicle detection. Key to the analysis is detecting people, vehicles, and objects of interest from frame-to-frame movements.

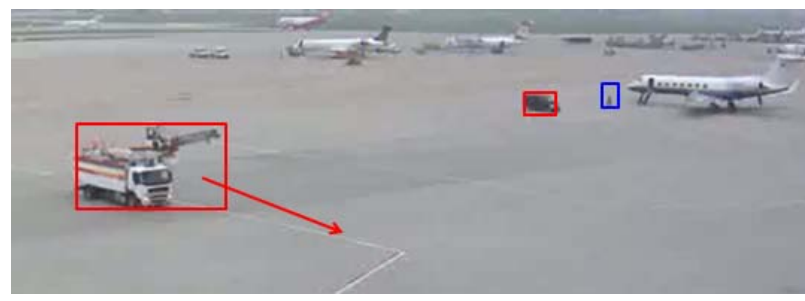

Figure 6. Airplane Parked and Object, Vehicle, and People Movement Detection

The movement indicates not only entities that are moving to key locations, but also normal and abnormal activity. Loading a plane, shown in Figure 7, would be a normal activity while abnormal activity would be people and objects moving on a runway.

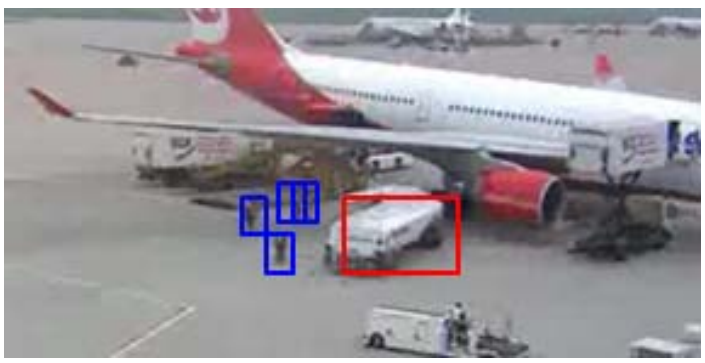

Figure 7. People Movement Detection (PersonPerson and Person-Object Interaction)

Abnormal activity can be detected to alert ATC operators of unsafe airport operations. In addition to Case 1 of UAV safe flight operations, ground surveillance can be used to monitor and detect interactions. Figures 8 and 9 show vehicles detected as well as the interactions of airport personnel.

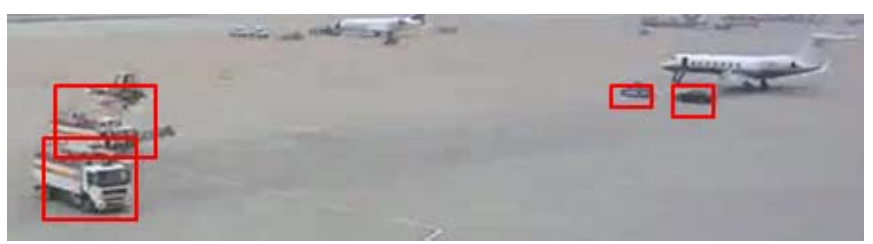

Figure 8. Vehicle-Environment Activity Detection

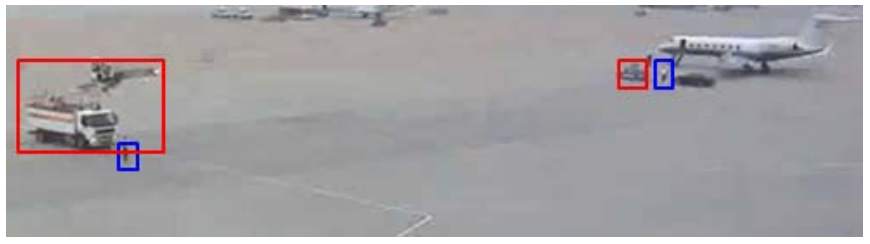

Figure 9. Person-Vehicle Activity Detection 


\section{Discussion and Conclusions}

Future NextGen air traffic control designs could include airport ground surveillance applications to facilitate unmanned aerial vehicle (UAV) and unmanned ground vehicle (UGV) operations. Building on our UAV/UGV developments [21, 25], this paper demonstrates L1 object tracker for activity analysis (e, g, person-object-vehicle-facility interaction) for automated airport ground surveillance. We focused on event triggering using video sensor networks that cues activity recognition from the airport camera datasets. As demonstrated for runway and terminal surveillance, automated video tracking could be used by air traffic controllers as additional alerts for safety of people, vehicles, and airplanes.

Future methods are being explored for UAV and UGV operations to support airport security, vehicle spacing, and user control. Given the context of a specified airport, we will combine the results with a User-Defined Operating Picture situation awareness display featured in our companion paper [98]. Finally, we will leverage our game-theoretical methods for monitoring activities from actors (e.g., UGVs) with abnormal behavior [99, 100].

\section{References}

[1] D. Pengfei, and M. U. De Haag, "A Simulation Environment for Evaluation of Integrated Alerting and Notification (IAN) Concepts," Proc. of the AIAA/IEEE Digital Avionics Systems Conf., 2012.

[2] R. Oliva, E. Blasch, and R. Ogan, "Applying Aerospace Technologies to Current Issues Using Systems Engineering, $3^{\text {rd }}$ Aess Chapter Summit," IEEE Aerospace and Electronic Systems Magazine, Vol. 28, No. 2, Feb. 2013.

[3] B. J. Glass, "Automated Data Exchange and Fusion for Airport Surface Traffic Management," Aiaa Guidance, Navigation, and Control Conf., 1997.

[4] E. P. Blasch, "Learning Attributes for Situational Awareness in the Landing of an Autonomous Aircraft," Proc. of the AIAA/IEEE Digital Avionics Conference, 1997.

[5] V. H. L. Cheng, "Collaborative Automation Systems for Enhancing Airport Surface Traffic Efficiency and Safety," Proc. of the AIAA/IEEE Digital Avionics Systems Conference, 2002.
[6] S. Atkins, C. Brinton, and D. Walton, "Functionalities, Displays, and Concept of Use for the Surface Management System," Proc. of the AIAA/IEEE Digital Avionics Systems Conference, 2002.

[7] V.L, Cheng, A. Yeh, and D.C. Foyle, "Evaluation Plan for an Airport Surface-Operation Automation Concept," Proc. of AIAA's Aviation Technology, Integration, and Operations (ATIO) Tech.Forum, 2003.

[8] D. R. Jones and L. J. Prinzle Iii, "Runway Incursion Prevention for General Aviation Operations," Digital Avionics Systems Conf., 2006.

[9] A. Guillonton, J-P. Arethens, C. Macabiau, a-C. Escher, D. Koeing, "State of the Art in Airport Navigation," Proc. of the AIAA/IEEE Digital Avionics Systems Conf., 2011.

[10] P. J. Smith, K. Weaver, A. B. Fermandes, Et Al., "Supporting Distributed Management of the Airport Surface," Proc. of the AIAA/IEEE Digital Avionics Systems Conf., 2012.

[11] S. Zelinski, "a Graph-Based Approach to Nominal Terminal Routing," Proc. of the AIAA/IEEE Digital Avionics Systems Conf., 2012.

[12] E. Blasch, Z. Wang, H. Ling, K. Palaniappan, G. Chen, D. Shen, A. Aved, Et. Al, "Video-Based Activity Analysis Using the L1 Tracker on Virat Data," IEEE Applied Imagery Pattern Rec. Workshop, 2013.

[13] E. Blasch, G. Seetharaman, K. Palaniappan, Et Al., "Wide-Area Motion Imagery (Wami) Exploitation Tools for Enhanced Situation Awareness," IEEE App. Imagery Patt. Rec. Workshop, 2012.

[14] E. Blasch "Introduction to Level 5 Fusion: the Role of the User," Chapter 19 in Handbook of Multisensor Data Fusion 2nd Ed, Eds. M. E. Liggins, D. Hall, and J. Llinas, Crc Press, 2008.

[15] E. P. Blasch, E. Bosse, and D. A. Lambert, High-Level Information Fusion Management and Systems Design, Artech House, 2012.

[16] E. Blasch, J. Salerno, S. J. Yang, L. Fenstermacher, I. Kadar, Et Al., "Summary of Human, Social, Cultural, Behavioral (HCSB) Modeling for Information Fusion," Proc. Spie, Vol. $8745,2013$. 
[17] E. Blasch, "Situation, Impact, and User Refinement," Proc. Spie, Vol. 5096, 2003.

[18] J. C. Klontz, A. K. Jain, "a Case Study on Unconstrained Facial Recognition Using the Boston Marathon Bombings Suspects," Technical Report Msu-Cse-13-4, 2013.

[19] E. Blasch, "Book Review: 3c Vision: Cues, Context, and Channels," IEEE Aerospace and Elect. Systems Mag., Vol. 28, No. 2, Feb. 2013.

[20] E. Blasch, "Level 5 (User Refinement) Issues Supporting Information Fusion Management" Int'l Conf. on Info Fusion, 2006.

[21] B. Liu, E. Blasch, Y. Chen, A. J. Aved, A. Hadiks, D. Shen, G. Chen, "Information Fusion in a Cloud Computing Era: a Systems-Level Perspective," Submitted to IEEE Aersopace and Elct. Sys. Mag, 2013.

[22] E. Blasch, A. Steinberg, S. Das, J. Llinas, C.-Y. Chong, O. Kessler, E. Waltz, and F. White, "Revisiting the Jdl Model for Information Exploitation," Int'l Conf. on Info Fusion, 2013.

[23] E. P. Blasch, D. A. Lambert, P. Valin, M. M. Kokar, J. Llinas, S. Das, C-Y. Chong, and E. Shahbazian, "High Level Information Fusion (HLIF) Survey of Models, Issues, and Grand Challenges," IEEE Aerospace and Electronic Systems Mag., Vol. 27, No. 9, Sept. 2012.

[24] E. Blasch, R. Breton, and P. Valin, "Using the C-Ooda Model for Cimic Analysis," Proc. IEEE Nat. Aerospace Electronics Conf., 2011

[25] B. Liu, Yu Chen, Et A1., "a Holistic CloudEnabled Robotics System for Real-Time Video Tracking Application," Future Information Technology, Vol. 276, Pp 455-468, 2014.

[26] P. C. G. Costa, Et Al., "Towards Unbiased Evaluation of Uncertainty Reasoning: the Urref Ontology," Int'l Conf. on Info Fusion, 2012.

[27] F. Bunyak, K. Palaniappan, S. K. Nath, G. Seetharaman, "Geodesic Active Contour-Based Fusion of Visible and Infrared Video for Persistent Object Tracking", IEEE Workshop on App. of Computer Vision, 2007.

[28] E. Blasch, "Proactive Decision Fusion for Site Security," Int'l Conf on Info. Fusion, 2005.
[29] E. Blasch, I. Kadar, J. Salerno, M. M. Kokar, S. Das, Et Al., "Issues and Challenges in Situation Assessment (Level 2 Fusion)," J. of Advances in Information Fusion, Vol. 1, No. 2, Pp. 122 - 139, Dec. 2006.

[30] E. Blasch, J. J. Salerno, and G. Tadda, "Measuring the Worthiness of Situation Assessment," Proc. IEEE Nat. Aerospace Elect.Conf, 2011.

[31] E. Blasch Et Al., "Dfig Level 5 (User Refinement) Issues Supporting Situational Assessment Reasoning," Int. Conf. on Info Fusion, 2005.

[32] S. J. Julier, J. K. Uhlmann, J. Walters, R. Mittu, K. Palaniappan, "the Challenge of Scalable and Distributed Fusion of Disparate Sources of Information," Proc. Spie, Vol. 6242, 2006.

[33] E. P. Blasch, D. A. Lambert, P. Valin, Et Al., "High Level Information Fusion (HLIF) Survey of Models, Issues, and Grand Challenges," IEEE Aerospace and Electronic Sys. Mag., Vol. 27, No. 9, Sept. 2012.

[34] E. Blasch, "Ontological Issues in Higher Levels of Information Fusion: User Refinement of the Fusion Process," Int. Conf. on Info Fusion, 2003.

[35] R. Carvalho, Et Al, "Prognos: Predictive Situational Awareness with Probabilistic Ontologies," Int'l Conf. on Info. Fusion, 2010.

[36] P.C.G. Costa, Kc Chang, Et Al., "High-Level Fusion: Issues in Developing a Formal Theory," Int'1 Conf. on Information Fusion, 2010.

[37] E. Blasch, P. Valin, E. Bossé, "Measures of Effectiveness for High-Level Fusion," Int'1 Conference on Information Fusion, 2010.

[38] A. Hoogs, J. Mundy, and G. Cross, "MultiModal Fusion for Video Understanding," IEEE Applied Imagery Pattern Rec. Workshop, 2001.

[39] A. Hoogs, J. Rittscher, G. Stein and J. Schmiederer, "Video Content Annotation Using Visual Analysis and a Large Semantic Knowledgebase,” IEEE Cvpr, 2003.

[40] M. T. Chan, A. Hoogs, J. Schmiederer, M. Petersen, "Detecting Rare Events in Video Using Semantic Primitives with Hmm," Int'l Conf. on Pattern Recognition, 2004. 
[41] M. T. Chan, A. Hoogs, Z. Sun, J. Schmiederer, R. Bhotika, and G. Doretto, "Event Recognition with Fragmented Object Tracks," Int'l. Conf. on Pattern Recognition, 2006.

[42] A. Basharat, A. Gritai, M. Shah, "Learning Object Motion Patterns for Anomaly Detection and Improved Object Detection," IEEE Cvpr, 2008.

[43] E. Swears, A. Hoogs, and A. G. A. Perera, "Learning Motion Patterns in Surveillance Video Using Hmm Clustering," IEEE Wmcv, 2008.

[44] E. Blasch, C. Banas, M. Paul, B. Bussjager, Et Al., "Pattern Activity Clustering and Evaluation (Pace)," Proc. Spie, Vol. 8402, 2012.

[45] K. K. Reddy, N. Cuntoor, Et A1., "Human Action Recognition in Large-Scale Datasets Using Histogram of Spatiotemporal Gradients," IEEE Int'1 Conf. on Adv. Video and Signal-Based Surveillance, 2012.

[46] P. Liang, Et Al., "Multiple Kernel Learning for Vehicle Detection in Wide Area Motion Imagery," Int. Conf. on Info Fusion, 2012.

[47] C. Yuan, X. Li, W. Hu, H. Ling, and S. Maybank, "3d R Transform on Spatio-Temporal Interest Points for Action Recognition," IEEE Conf. on Computer Vision and Pattern Recognition (Cvpr), 2013.

[48] J. Gao, H. Ling, Et Al., "Pattern of Life from Wami Objects Tracking Based on Visual ContextAware Tracking and Infusion Network Models," Proc. Spie, Vol. 8745, 2013.

[49] D. Ramanan, D.A. Forsyth, A. Zisserman, "Tracking People by Learning Their Appearance," IEEE Pattern Analysis and Machine Intelligence, 29(1):65-81, 2007.

[50] P. Turaga, R. Chellappa, V. S. Subrahmanian, and O. Udrea, "Machine Recognition of Human Activities: a Survey," IEEE Trans. on Circuits and Systems for Video Technology, Vol. 18, No. 11, Nov. 2008.

[51] E. Blasch, Derivation of a Belief Filter for High Range Resolution Radar Simultaneous Target Tracking and Identification, Ph.D. Dissertation, Wright State University, 1999.

[52] T. Wang, Z. Zhu and R. Hammoud, "AudioVisual Feature Fusion for Vehicles Classification in a
Surveillance System," IEEE Conf. on Computer Vision and Pattern Recognition (Cvpr), 2013.

[53] I. Kim, S. Oh, B. Byun, A. G. A. Perera, C-H. Lee, "Explicit Performance Metric Optimization for Fusion-Based Video Retrieval," European Conference on Computer Vision (Eccv), 2012.

[54] B. Kahler and E. Blasch, "Sensor Management Fusion Using Operating Conditions," Proc. IEEE Nat. Aerospace Electronics Conf, 2008.

[55] J. Garcia Herro, L. Snidaro, and I. Visentini, "Exploiting Context as a Binding Element for MultiLevel Fusion," Int'l Conf. on Info. Fusion, 2012.

[56] E. P. Blasch and P. Hanselman, "Information Fusion for Information Superiority," IEEE Nat. Aerospace and Electronics Conference, 2000.

[57] E. Blasch, "Sensor, User, Mission (Sum) Resource Management and Their Interaction with Level 2/3 Fusion" Int'l Conf. on Info Fusion, 2006.

[58] E. Blasch, I. Kadar, K. Hintz, Et Al., "Resource Management Coordination with Level 2/3 Fusion Issues and Challenges," IEEE Aero. and Elec. Sys. Mag., Vol. 23, No. 3, Pp. 32-46, Mar. 2008.

[59] M. S. Beigi, S. Calo, and D. Verma, "Policy Transformation Techniques in Policy-Based Systems Management," IEEE Int'l. Workshop on Policies for Distributed Systems and Networks, 2004.

[60] E. Blasch and B. Kahler, "Multi-Resolution Eo/Ir Tracking and Identification," Int'l Conf. on Info Fusion, 2005.

[61] M. H. Kolekar, Et Al., "Event Detection and Semantic Identification Using Bayesian Belief Networks," IEEE Video-Orientation. Object and Event. Classification, 2009.

[62] Y. Yin, H. Man, J. Wang, and G. Yang, "Human Motion Change Detection by Hierarchical Gaussian Process Dynamical Model with Particle Filter." IEEE Audio-Vis Sys Surveillance, 2010.

[63] G. Levchuk, A. Bobick, and E. Jones, E., "Activity and Function Recognition for Moving and Static Objects in Urban Environments from WideArea Persistent Surveillance Inputs," Proc. Spie, Vol. 7704, 2010.

[64] Z. Yao, X. Yang, L. Lin, M. W. Lee, Et Al.., "I2t: Image Parsing to Text Description," Proc. of IEEE, Vol. 98, No.8, Pp 1485-1508, 2010. 
[65] Y. Wu, E. Blasch, G. Chen, L. Bai, and H. Ling, "Multiple Source Data Fusion Via Sparse Representation for Robust Visual Tracking," Int. Conf. on Info Fusion, 2011.

[66] Y. Wu, H. Ling, E. Blasch, G. Chen, and L. Bai, "Visual Tracking Based on Log-Euclidean Riemannian Sparse Representation," Int. Symp. on Adv. in Visual Computing, 2011.

[67] E. Blasch, H. Ling, Y. Wu, Et Al., "Dismount Tracking and Identification from Electro-Optical Imagery," Proc. Spie, Vol. 8402, 2012.

[68] Y. Wu, J. Wang, J. Cheng, H. Lu, Et Al., "RealTime Probabilistic Covariance Tracking with Efficient Model Update," IEEE Trans. on Image Processing, 21(5):2824-2837, 2012.

[69] G. Seetharaman, G. Gasperas, and K. Palaniappan, "a Piecewise Affine Model for Image Registration in 3-D Motion Analysis," IEEE Int. Conf. Image Processing, Pp. 561-564, 2000.

[70] A. Hafiane, K. Palaniappan, G. Seetharaman, "Uav-Video Registration Using Block-Based Features," IEEE Int. Geoscience and Remote Sensing Symposium, Vol. Ii, Pp. 1104-1107, 2008.

[71] E. P. Blasch and T. Connare, "Group Tracking of Occluded Targets," Proc. Spie, Vol. 4365, April 2001.

[72] E. Blasch and T. Connare, "Improving Track Maintenance Through Group Tracking," Proc. of the Workshop on Estimation, Tracking, and Fusion; a Tribute to Yaakov Bar Shalom, 360 -371, May 2001.

[73] T. Connare, E. Blasch, Et Al., "Group Imm Tracking Utilizing Track and Identification Fusion," Proc. of the Workshop on Estimation, Tracking, and Fusion; a Tribute to Yaakov Bar Shalom, May 2001.

[74] C. Yang and E. Blasch, "Fusion of Tracks with Road Constraints," J. of. Advances in Information Fusion, Vol. 3, No. 1, 14-32, June 2008.

[75] X. Shi, H. Ling, E. Blasch, and W. Hu, "Context-Driven Moving Vehicle Detection in Wide Area Motion Imagery," Int'l Conf on Pattern Recognition (ICPR), 2012.

[76] S. Candemir, K. Palaniappan, F. Bunyak, G. Seetharaman, R.M. Rao, "Feature Prominence-Based Weighting Scheme for Video Tracking", Indian
Conf. Computer Vision, Graphics and Image Processing, 2012.

[77] M. Poostchi, F. Bunyak, Et Al., "Feature Selection for Appearance-Based Vehicle Tracking in Geospatial Video," Proc. Spie, 2013.

[78] E. D. Marti, J. Garcia, and J. L Crassidis, "Improving Multiple-Model Context-Aided Tracking Through an Autocorrelation Approach," Int'l Conf. on Info Fusion, 2012.

[79] R. Pelapur, K. Palaniappan, G. Seetharaman, "Robust Orientation and Appearance Adaptation for Wide-Area Large Format Video Object Tracking", IEEE Int. Conf. Adv. Video and Signal-Based Surv., 2012.

[80] F. Bunyak, K. Palaniappan, S. K. Nath, G. Seetharaman, "Flux Tensor Constrained Geodesic Active Contours with Sensor Fusion for Persistent Object Tracking," J .of Multimedia, Vol. 2, No. 4, Pp. 20-33, Aug, 2007.

[81] R. Pelapur, S. Candemir, F. Bunyak, M. Poostchi, Et. Al, "Persistent Target Tracking Using Likelihood Fusion in Wide-Area and Full Motion Video Sequences," Int'l Conf. on Info. Fusion, 2012.

[82] I. Ersoy, K. Palaniappan, G. Seetharaman, "Visual Tracking with Robust Target Localization," IEEE Int. Conf. Image Processing, 2012.

[83] P. Liang, Et Al., "Multiple Kernel Learning for Vehicle Detection in Wide Area Motion Imagery," Int. Conf. on Info Fusion, 2012.

[84] X. Shi, H. Ling, E. Blasch, and W. Hu, "Context-Driven Moving Vehicle Detection in Wide Area Motion Imagery," Int'l Conf on Pattern Recognition (Icpr), 2012.

[85] P. Liang, H. Ling, E. Blasch, G. Seetharaman, D. Shen, G. Chen, "Vehicle Detection in Wide Area Aerial Surveillance Using Temporal Context," Int'1 Conf. on Info Fusion, 2013.

[86] X. Shi, P. Li, W. Hu, E. Blasch, H. Ling, "Using Maximum Consistency Context for Multiple Target Association in Wide Area Traffic Scenes," Int'l Conf. on Acoustics, Speech and Signal Processing (Icassp), 2013.

[87] C. Yang and E. Blasch, "Kalman Filtering with Nonlinear State Constraints," IEEE Tr. Aero. and Elec. Sys., Vol. 45 (1): 70-84, 2009. 
[88] Z. Duan, X. Rong Li, Et Al., "Design and Analysis of Linear Equality Constrained Dynamic Systems," Int'1 Conf. on Info. Fusion, 2012.

[89] O. Straka, J. Dunık, M. Šimandl, Et Al., "Randomized Unscented Transform in State Estimation of Non-Gaussian Systems: Algorithms and Performance," Int. Conf. on Info Fusion, 2012.

[90] H. Ling, L. Bai, Et Al., "Robust Infrared Vehicle Tracking Across Target Pose Change Using L1 Regularization," Int'l Conf. on Info. Fusion, 2010.

[91] X. Mei, H. Ling, Et Al., "Minimum Error Bounded Efficient L1 Tracker with Occlusion Detection," IEEE Comp. Vision and Pattern Rec., 2011.

[92] X. Zhang, W. Li, W. Hu, H. Ling, Et Al., "Block Covariance Based L1 Tracker with a Subtle Template Dictionary," Pattern Recognition, 2012.

[93] X. Mei, H. Ling, Y. Wu, Et Al., "Efficient Minimum Error Bounded Particle Resampling L1 Tracker with Occlusion Detection," IEEE Trans. on Image Processing (T-Ip), Vol. 22 (7), 2661 - 2675, 2013.

[94] K. Palaniappan, F. Bunyak, P. Kumar, Et Al., "Efficient Feature Extraction and Likelihood Fusion for Vehicle Tracking in Low Frame Rate Airborne Video," Int'l Conf. on Information Fusion, 2010.
[95] Kaushik, "Strange Airport: Gibraltar, World's Only Airport Runway Intersecting a Road," Amusing Planet, Nov 3, 2011. Accessed at http://www.amusingplanet.com/2011/11/gibraltarairport-worlds-only-airport.html

[96] Gibraltar airport video: www.youtube.com/watch?v=MTnWIj7_CyY

[97]

www.webcamgalore.com/EN/webcam/Germany/Stut tgart/182.html

[98] E. Blasch, "Enhanced Air Operations Using Jview for an Air-Ground Fused Situation Awareness Udop," AIAA/IEEE Digital Avionics Systems Conference, Syracuse, Ny, Oct. 2013.

[99] D. Shen, G. Chen, J. Cruz, and E. Blasch, "a Game Theoretic Data Fusion Aided Path Planning Approach for Cooperative Uav Control," IEEE Aerospace Conf., 2008.

[100] D. Shen, G. Chen, H. Ling, K. Pham, and E. Blasch, "A Robot Demonstrator Design for TwoPlayer Pursuit-Evasion Games," Submitted to Decision Support Systems, 2013.

33rd Digital Avionics Systems Conference October 5-9, 2014 